\section{Factors improving teachers' self-efficacy for conducting SST -to improve work engagement of teachers' -}

Kenichi KUKIYAMA, Yayoi ITO \& Yuko YAMAGUCHI (Kyushu Sangyo University, JAPAN)

\section{Backgrounds and Objectives:}

Work engagement is defined as "a positive work-related condition that is characterized by vigor, dedication, and absorption, which is a general affective-cognitive state, and not a momentary state" (Schaufeli et al., 2002) Work engagement scores of Japanese are low (Shimizu et al., 2010), which is especially the case of teachers that are exhausted (Takagi, 2010). In 2010, MEXT advocated Social Skills Training (SST) as the core of new educational consultation techniques.

However, it has been rarely conducted in Japan to date. Even when SST is undertaken, it is often conducted by specialists. It has been suggested that teachers might develop confidence if they conducted SST by themselves. This was also expected to increase their satisfaction with work and prevent burnout (Skaalvik, E. M.\& Skaalvik, S., 2009), which could result in improvements to their work engagement This study analyzed factors improving teachers' self-efficacy for conducting SST.

\section{Methods:}

A questionnaire assessing "Images of SST" and "Images of executing SST" was administered to in-service teachers $(n=175)$ working in Japanese kindergartens, elementary schools, as well as junior and senior high schools.

\section{Results:}

The results of factor analysis indicated four sub-scales of teachers' images of SST: This could be a place for your sources. "significant," "stereotyping," "expectations of results," and "personality development." Moreover, four sub-scales of teachers' images of executing SST; "motivation," "execution possibilities at schools," "realistic estimates for preparation," and "self-efficacy for execution."

Scores of SST images were high in both SST-experienced and SST-inexperienced groups. Moreover, a positive correlation was shown between SST images and images of SST execution. The scores of estimates for preparation and self-efficacy for execution were high in images of SST execution in the experienced group $(p<.01)$

\section{Discussion:}

Teachers had positive images of SST regardless of experience. Moreover, images of SST and images of executing SST were positively correlated. It is considered that negative SST images were not obstacles to conducting SST.

Teachers having experienced SST could make realistic estimates of preparation and had high self-efficacy for execution. Experience of SST was a factor in improving the self-efficacy for conducting SST. Therefore, it is suggested that SST in which busy teachers could easily participate should be developed. It is concluded that such SST would improve the work engagement of teachers.

This work was supported by JSPS KAKENHI Grant Number JP18K02879.
Table 1 Factor analysis of teachers' images of STT

\begin{tabular}{|c|c|c|c|c|c|c|c|c|c|}
\hline \multirow{2}{*}{$\begin{array}{l}\text { effective } \\
\text { helpful for students }\end{array}$} & & $\frac{2}{.11}$ & \multicolumn{2}{|c|}{$\begin{array}{ll}3 & 4 \\
\end{array}$} & \multicolumn{3}{|c|}{$\begin{array}{c}\text { Table } 2 \text { Factor analysis of teachers' images of } \\
\text { executing SST }\end{array}$} & & \\
\hline & .84 & -.01 & -.02 & .09 & & 1 & 2 & & \\
\hline meaningful & .70 & -.03 & -.02 & .09 & 19 willing to receive trainings & 87 & 13 & -.14 & $\overline{-.14}$ \\
\hline effective to education & & & & & 11 willing to implement SST by myself & .73 & -.03 & .11 & -.08 \\
\hline & & 01 & & -.08 & 8 willing to study SST by books or online & 61 & -.29 & -.02 & .15 \\
\hline availability & .61 & -.04 & 23 & -.03 & 15 willing to become an SST trainer & .58 & -.09 & .22 & 16 \\
\hline discouraging individuality & -.09 & .80 & 16 & -.03 & 18 having a natural ability to be an SST trainer & 49 & -.05 & .37 & 10 \\
\hline making a passive personality & .09 & .79 & -.03 & -.05 & 20 confident to implement SST & 42 & 16 & .39 & -.04 \\
\hline compulsion of values & & 70 & .00 & .00 & 4 confident to be understood by the & -.21 & .77 & .03 & -.08 \\
\hline & & 10 & (I & .04 & 14 able to get help by colleagues & -.11 & .71 & .20 & -.07 \\
\hline making a puppet-like personality & -.04 & .77 & .02 & -.06 & $17 \mathrm{SS}$ & .05 & .55 & .07 & -.17 \\
\hline ficial changes & .08 & 40 & -39 & .05 & es to implement SST & . 12 & .53 & -28 & . 38 \\
\hline not helfful for students & -28 & .35 & -18 & .05 & 3 having & .06 & .41 & -.07 & 31 \\
\hline making deep changes & -.06 & .08 & 63 & .43 & $\begin{array}{l}12 \mathrm{~h} \\
16 \mathrm{~h}\end{array}$ & .26 & 34 & .17 & $\begin{array}{r}.09 \\
-29\end{array}$ \\
\hline leading to good results if it is done & .24 & .05 & 60 & .03 & 13 confid & .11 & .08 & .75 & .03 \\
\hline no changes even if it is done & 10 & -.15 & .60 & -12 & asible if trained & .03 & .01 & .68 & -.01 \\
\hline changing interpersonal relationship & 21 & .05 & .56 & -05 & 9 having an opportunity to learn SST $s$ & -.03 & -.01 & .19 & .58 \\
\hline & & & & & 5 being equipped to implement SST & -.07 & -.07 & .31 & .55 \\
\hline & -.11 & .00 & .17 & .83 & learn how to run & 10 & .11 & -.08 & .53 \\
\hline utilizing personal values & 28 & -.10 & -.25 & 61 & 2 preparations for SST sounds tough & 22 & -.10 & -.14 & 46 \\
\hline 1 & & -.37 & 59 & 43 & 6 having training is necessary to implement $\mathrm{SS}^{-}$ & -.40 & -.03 & .23 & 43 \\
\hline 2 & & & -.41 & -20 & & & 46 & 48 & .35 \\
\hline 3 & & & & .40 & 3 & & & & 39 \\
\hline
\end{tabular}

Table 3 Correlations between measures

\begin{tabular}{|c|c|c|c|c|c|c|c|}
\hline & stereotyping & $\begin{array}{l}\text { expectations } \\
\text { of results }\end{array}$ & $\begin{array}{l}\text { personality } \\
\text { development }\end{array}$ & motivation & $\begin{array}{c}\text { implementation } \\
\text { possibilities at } \\
\text { schools }\end{array}$ & $\begin{array}{l}\text { self-efficacy for } \\
\text { implementation }\end{array}$ & $\begin{array}{c}\text { realistic } \\
\text { estimation for } \\
\text { preparation }\end{array}$ \\
\hline $\begin{array}{l}\text { significant } \\
\text { stereotyping } \\
\text { expectations of results } \\
\text { personality development } \\
\text { motivation } \\
\text { implementation possibilities at schools } \\
\text { self-efficacy for implementation } \\
\end{array}$ & $-.31^{\star \star}$ & $\begin{array}{r}.65^{* *} \\
-.36^{* *}\end{array}$ & $\begin{array}{r}.44^{* *} \\
-.23^{* *} \\
.42^{* *}\end{array}$ & $\begin{array}{l}.36^{* *} \\
-.14 \\
.34^{* *} \\
.16^{*}\end{array}$ & $\begin{array}{c}.23^{* *} \\
-.09^{* *} \\
.28^{* *} \\
-.03 \\
.35^{* *}\end{array}$ & $\begin{array}{l}.18^{*} \\
-.13 \\
.24^{* *} \\
.09 \\
.58^{* *} \\
.22^{* *}\end{array}$ & $\begin{array}{l}.08 \\
.05 \\
.02 \\
.10 \\
.37^{* *} \\
.37^{* *} \\
.39^{* *} \\
\end{array}$ \\
\hline
\end{tabular}

Table 4 Means and standard deviations of sub-sample

\begin{tabular}{|c|c|c|c|c|c|c|c|}
\hline & \multicolumn{3}{|c|}{ experienced } & \multicolumn{3}{|c|}{ inexperienced } & \multirow{2}{*}{$\mathrm{t}$ value } \\
\hline & MEAN & $S D$ & $n$ & MEAN & $S D$ & $n$ & \\
\hline significant & 4.55 & 0.53 & 17 & 4.25 & 0.59 & 122 & $2.03 *$ \\
\hline stereotyping & 1.95 & 1.02 & 16 & 2.27 & 0.66 & 122 & -1.2 \\
\hline expectations of results & 3.75 & 0.67 & 17 & 3.64 & 0.58 & 124 & 0.75 \\
\hline personality development & 3.50 & 0.77 & 17 & 3.60 & 0.66 & 124 & -0.6 \\
\hline motivation & 3.46 & 0.65 & 16 & 3.03 & 0.67 & 122 & 2.44 * \\
\hline implementation possibilities at schools & 3.04 & 0.62 & 17 & 2.76 & 0.68 & 122 & 1.59 \\
\hline self-efficacy for implementation & 3.41 & 0.89 & 17 & 2.78 & 0.80 & 123 & $2.98^{* *}$ \\
\hline realistic estimation for preparation & 2.33 & 0.62 & 17 & 1.83 & 0.51 & 123 & $3.67^{* *}$ \\
\hline
\end{tabular}

